\title{
A descriptive survey of porcine epidemic diarrhea in pig populations in northern Vietnam
}

\author{
Thi Ngan Mai ${ }^{1,2} \cdot$ Wataru Yamazaki ${ }^{3} \cdot$ Thanh Phong Bui $^{4} \cdot$ Van Giap Nguyen ${ }^{2} \cdot$ Thi My Le Huynh $^{2} \cdot$ Shuya Mitoma ${ }^{1} \cdot$ \\ Hala El Daous ${ }^{1,5} \cdot$ Emmanuel Kabali $^{6} \cdot$ Junzo Norimine $^{7,8} \cdot$ Satoshi Sekiguchi $^{7,8}$ (I)
}

Received: 8 April 2020 / Accepted: 24 September 2020 / Published online: 4 October 2020

(C) Springer Nature B.V. 2020

\begin{abstract}
Porcine epidemic diarrhea (PED) virus (PEDV) is a globally emerging and re-emerging epizootic swine virus that causes massive economic losses in the swine industry, with high mortality in piglets. In Vietnam, PED first emerged in 2009 and has now developed to an endemic stage. This is the first cross-sectional survey performed to evaluate the proportion of PEDV-positive swine farms in Vietnam from January 2018 to February 2019. Fecal samples from 327 pig farms in northern Vietnam were collected and tested for PEDV infection by reverse transcription-loop-mediated isothermal amplification (RT-LAMP) method. The proportion of PEDV-positive farms was 30.9\% and PEDV-positive farms were distributed throughout the study area. The highest proportion of PEDV-positive farms was 70\% (7/10) among nucleus production type farms $(P<0.05)$. Higher proportions of PEDV-positive farms were found in the Northeast and Red River Delta areas, which are the major areas of pig production $(P<0.05)$. The proportion of PEDV-positive farms was higher among larger farms $(P<0.05)$. Our findings illustrate the high proportion of PEDV-positive farms in the Vietnamese pig population and will help to better understand the epidemiological dynamics of PED infection, to estimate impact, and establish and improve prevention and control measures.
\end{abstract}

Keywords PEDV $\cdot$ Pooled sample $\cdot$ Descriptive survey $\cdot$ Cross-sectional study $\cdot$ Vietnam

\section{Introduction}

Economic impacts of transboundary animal diseases (TADs), which include impacts on production, food security, trade, and environment. Porcine epidemic diarrhea (PED) is one of the TADs, worldwide emerging and re-emerging epizootic swine disease. The global re-emergence of PED requires a deeper understanding of the dynamics of porcine epidemic diarrhea

Electronic supplementary material The online version of this article (https://doi.org/10.1007/s11250-020-02416-1) contains supplementary material, which is available to authorized users.

Satoshi Sekiguchi

sekiguchi@cc.miyazaki-u.ac.jp

1 Graduate School of Medicine and Veterinary Medicine, University of Miyazaki, Miyazaki, Japan

2 Faculty of Veterinary Medicine, Vietnam National University of Agriculture, Hanoi, Vietnam

3 Center for Southeast Asian Studies, Kyoto University, Kyoto, Japan

4 MNS Farm Nghe An, Masan Nutri-Science, Masan Group, Quy Hop, Nghe An, Vietnam virus (PEDV) spread in the world. This study focused on the current epidemiology of PEDV in Vietnam. Our results will encourage stakeholders in swine industry to implement preventive measures for PEDV control appropriately. PEDV is the causative agent of PED. PED is a devastating disease characterized by acute enteritis, vomiting, and severe watery diarrhea and imposes massive economic losses in the swine industry with high morbidity and mortality in piglets (Song

5 Faculty of Veterinary Medicine, Benha University, Moshtohor, Toukh, Qalyubia, Egypt

6 Quality Assurance Unit, Zambia Medicines Regulatory Authority, Lusaka, Zambia

7 Department of Veterinary Science, Faculty of Agriculture, University of Miyazaki, Miyazaki, Japan

8 Center for Animal Disease Control, University of Miyazaki, Miyazaki, Japan 
and Park 2012; Lee 2015). PEDV was first observed in England in 1971 and was later reported in European countries such as Belgium, England, Germany, and France (Wood 1977; Pensaert and De Bouck 1978). Since the 1980s, the most intense outbreaks have mainly occurred in the swine industries of Asian countries including Korea, China, Japan, Philippines, and Thailand (Takahashi et al. 1983; Song and Park 2012). More recently, a highly pathogenic PEDV was detected in early 2013 in the United States (US). This PEDV strain resulted in high morbidity and mortality, dramatically affecting US swine production, and transmitted further into neighboring countries (Canada and Mexico) (Kochhar 2014; Lee 2015; Niederwerder and Hesse 2018). Subsequently, large-scale PED outbreaks recurred in Japan, South Korea, and Taiwan (Lin et al. 2014; Lee 2015; Toyomaki et al. 2018).

In Vietnam, pork is one of the most important agricultural products, contributing $58 \%$ of the total agricultural gross domestic product (Dietze 2011). PED was first observed in the southern provinces in 2009 (Do et al. 2011). Previous studies demonstrated that the Vietnamese PEDV strains currently circulating are new variants closely related to Chinese strains and provided evidence of disease spread throughout the major swine-producing regions (Do et al. 2011; Vui et al. 2014; Kim et al. 2015; Vui et al. 2015; Choe et al. 2016; Diep et al. 2018). Northern Vietnam has a long border with southern China, with pigs being transported to China and transportation vehicles returning daily. The proportion of PEDV-positive farms is high $(41 \%)$ in southern China, which could represent a potential risk for transmission of PEDV to Vietnam (Chen et al. 2019). PED has now developed to an endemic stage and continues to cause economic damage for the swine industry in Vietnam (Lee 2015; Vui et al. 2015; Diep et al. 2018). However, reporting of PEDV infection to local and national veterinary organizations is not required. Thus, it is crucial to better understand the current epidemiology of PEDV in Vietnam to appropriately implement control programs. The purpose of this study was therefore to assess the current farmlevel proportion of PEDV-positive farms and to analyze the basic epidemiological features of PEDV infection in Vietnam.

\section{Materials and methods}

\section{Study area and design}

Vietnam is divided into eight ecological zones (Northeast, Northwest, Red River Delta, North Central Coast, Central Highlands, South Central Coast, Southeast, and Mekong River Delta) based on similarities in geographical features and climate, as defined by the General Statistics Office of Vietnam. In our study, a stratified sampling method was applied based on the density of pig population. Samples from 327 voluntary farms including backyard farms were collected between January 2018 and February 2019 from the north (Northeast, Northwest, and Red River Delta) and central (North Central Coast) regions of Vietnam where the largest numbers of pigs are located (approximately $60 \%$ of all pig heads in Vietnam; http://channuoivietnam.com/thong-kechan-nuoi/) (Fig. 1a) and the number of farms is about 2 million farms (including private and company farms). Farms were defined as one or more buildings located in close geographical proximity under the same ownership with animals managed as a single population. Within each herd, the number of samples required for detection of PEDV was determined according to within-herd prevalence data obtained in China (Wang et al. 2016). The required sample size was estimated as 20 samples per farm. Therefore, on each farm, at least 20 samples were collected from pigs showing clinical signs of PED, pigs showing any signs of diarrhea or healthy animals (Supplementary Data 1). Samples from all pigs were collected on farms with pig herds of less than 20 animals. Onfarm, fecal samples were collected into tubes or plastic bags. In total, 6601 fecal samples were collected from 327 farms, comprising private farms and six industrial swine farms, located in 19 provinces in northern Vietnam. For each of the 327 sites, data were available regarding location (commune, district, and province), farm size, production type (farrow-to-finish, farrow-to-wean, nucleus, wean-to-finish (Thomas et al. 2015)), farm identification, and the collection or receipt date of fecal samples. It has been confirmed that other enteric viruses including swine acute diarrhea syndrome coronavirus (SADS-CoV) and transmissible gastroenteritis virus (TGEV) have never been detected by monitoring in this study area.

\section{Pooled samples}

The sensitivity of the pooled testing system indicated that a pool of up to 15 individual fecal samples could be analyzed (Mai et al. 2018). In addition, based on preliminary data, we confirmed the efficiency of the pooled testing system for identification of PEDV-infected herds (Supplementary Data 2). Thus, we collected up to 20 individual samples from each farm and combined these into two pooled samples for test. All samples were collected in ice boxes, transported on dry ice, and stored at $-20^{\circ} \mathrm{C}$ until use. Briefly, fecal samples were diluted in phosphate-buffered saline and centrifuged at $2300 \times g$ at $4{ }^{\circ} \mathrm{C}$ for $10 \mathrm{~min}$ as previously described (Mai et al. 2018). After centrifugation, $100 \mu \mathrm{L}$ of supernatant from each pooled sample (10 animals each) were transferred to a new tube. Finally, the remaining $1 \mathrm{~mL}$ of each pooled sample was stored for future use.

\section{Nucleic acid extraction}

Nucleic acid was extracted from all pooled samples from the 327 farms using the taco ${ }^{\mathrm{TM}}$ DNA/RNA Extraction Kit 
Fig. 1 Spatial distribution of pigs in Vietnam and the geographical locations of pig farms in northern Vietnam. a Pig density is illustrated by deepening shades of green, indicating category level; square indicates the study area. b Digital elevation model of the location of pig farms in northern Vietnam. A total of 6601 samples were collected on 327 pig farms located in 19 provinces in northern Vietnam from January 2018 to February 2019. Red dots indicate PEDV-positive farms and blue dots indicate PEDVnegative farms

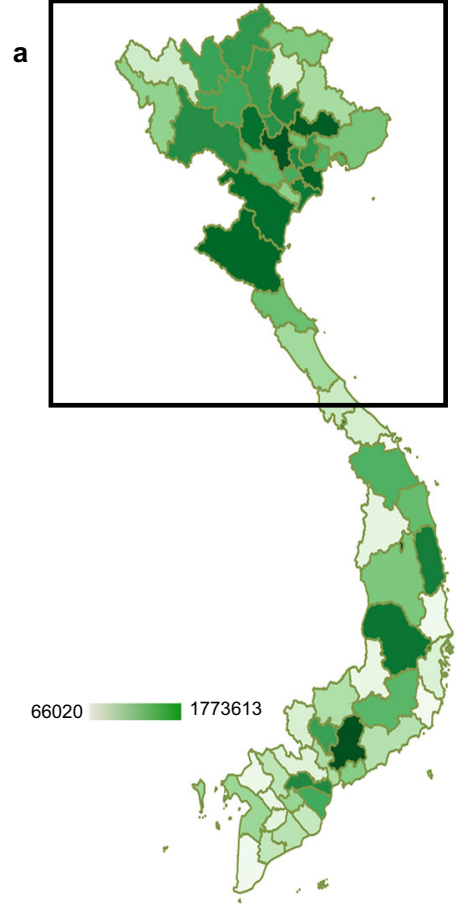

(GeneReach Biotech, Taichung City 407, Taiwan) and the taco $^{\text {TM }}$ mini Nucleic Acid Automatic Extraction System (ta$\mathrm{Co}^{\mathrm{TM}}$ mini; Gene Reach Biotech) in accordance with the manufacturer's instructions. Nucleic acids were eluted in $50 \mu \mathrm{L}$ of elution buffer, transferred to fresh tubes, and stored at $-80{ }^{\circ} \mathrm{C}$ for later use.

\section{Diagnostic test methods}

We used reverse transcription-loop-mediated isothermal amplification (RT-LAMP) with a colori-fluorometric indicator (CFI) to detect PEDV infection as described previously (Hayashida et al. 2015; Mai et al. 2018). The results of our previous study indicated that the accuracy of the RT-LAMP method was comparable with RT-PCR as the standard diagnostic method and 100 times more sensitive (Mai et al. 2018). As an indicator for the LAMP reaction, we developed a combination of dyes that we designated the CFI. The CFI stock solution consisted of $3 \mathrm{mM}$ hydroxynaphthol blue (HNB, MP Biomedicals, Aurora, $\mathrm{OH})$ and $0.35 \%$ v/v GelGreen $(10,000 \times$ solution, Biotium, Hayward, CA) dissolved in distilled water. The CFI-RT-LAMP reaction was conducted in a final volume of $25 \mu \mathrm{L}$ comprising $2 \mu \mathrm{L}$ of RNA template, the inner primer pair $(1.6 \mu \mathrm{M}$ each), the loop primer pair $(0.8 \mu \mathrm{M}$ each), the outer primer pair $(0.2 \mu \mathrm{M}$ each $), 1.6 \mathrm{mM}$ dNTPs, $5 \mathrm{M}$ betaine, $20 \mathrm{mM}$ Tris- $\mathrm{HCl}$ (pH 8.8), $10 \mathrm{mM} \mathrm{KCl}, 10 \mathrm{mM}$ (NH4)2SO4, $4 \mathrm{mM} \mathrm{MgSO} 4,8 \mathrm{U}$ of Bst DNA polymerase (New England Biolabs, Ipswich, MA), $0.15 \mathrm{U}$ of AMV reverse transcriptase (Invitrogen, Carlsbad, CA), and $1 \mu \mathrm{L}$ of CFI stock solution to allow visual detection. Amplification reactions were performed at $63{ }^{\circ} \mathrm{C}$ for $40 \mathrm{~min}$, then terminated by heating at $80^{\circ} \mathrm{C}$. All pooled samples were tested using the CFI-RTLAMP assay to detect PEDV. Swine herds with at least one confirmed positive pooled sample were defined as PEDVpositive farms for the purposes of this study.

\section{Data analysis}

Descriptive statistics were computed for all variables. To investigate associations between the proportion of PEDV-positive farms and each variable, pairwise comparisons were made using the chi-squared test with the pairwise.prop.test function and $P$ values were adjusted using the Bonferroni method. Ninety-five percent confidence intervals (95\% CIs) were obtained from parameter estimates and $P$ values $<0.05$ were considered statistically significant. We used the glm package in $\mathrm{R}$ to analyze the fitting generalized linear models in the multiple logistic regression analysis. Backwards stepwise variable selection was performed, beginning with the least significant variable. After the main effects were identified, all possible twoway interactions were also examined. The final model was obtained with $P$ levels for the remaining variables of $<0.05$. The strengths of association between all the variables and PEDV pig farm status by evaluating the odds ratios (ORs) and the corresponding CIs. All analyses were conducted using R (version 3.4.3, Vienna, Austria).

The geographical locations of farms were mapped using a free and open source Quantum Geographic Information System (QGIS) version 2.14.14 (https://www.qgis.org/en/ site/). Spatial statistics were used to evaluate the spatial 
distributions of pig farms by using analysis tools with nearest neighbor statistics in QGIS. The expected average distance is the average distance between farms in a hypothetical random distribution while the observed mean distance is based on the actual average distance from each farm to its nearest neighboring farm. The nearest neighbor index is used to evaluate the spatial distribution of pig farms whether they are clustered or dispersed. If the index is less than 1 , the pattern exhibits clustering. If the index is greater than 1 , the trend is toward dispersion. Z-scores are standard deviations that very high or very low (negative) $z$-scores, associated with very small $P$ values. The average distance to the nearest neighboring farm was calculated using the nearest neighbor analysis tool in QGIS. Using this tool, geographical data were returned as four values: observed mean distance, expected mean distance, nearest neighbor index, and Z-score. The nearest neighbor index is expressed as the ratio of the observed mean distance to the expected mean distance. The Z-score calculates a standardized difference between the observed and expected value of a statistic.

\section{Results}

In total, PEDV was detected on 101 (30.89\%) farms during the study period (Table 1). Out of 101 PEDV-positive farms, $28.71 \%$ (29/101) were affected by PED with animals showing clinical signs while more than $70 \%(72 / 101)$ had subclinically infected animals. We compared the proportion of PEDVpositive farms by stratifying by different variables including farm status, production type, area, and farm size. The results showed statistically significant differences in PEDV positivity based on production type, area, and farm size; however, the proportion of PEDV-positive farms was not associated with farm status (company vs. private farms). The proportion of PEDV-positive farms in nucleus production type farms $(70.0 \%)$ was higher than those in farrow-to-wean production type farms $(23.9 \% ; P<0.05)$. However, the number of nucleus farms in this study was limited (10 farms). Moreover, a higher proportion of PEDV-positive farms was observed in the Northeast and Red River Delta regions $(P<0.05)$. The proportion of PEDV-positive farms was higher among large farms $(\geq 600$ animals included sows and/or fattening pigs) than in small farms $(<600$ animals included sows and/or fattening pigs) $(P<0.05$; Table 1). The statistics (minimum, 1 st quantile, median, mean, 3rd quantile, maximum) of farm size were $15.0,200.0,600.0,870.8,1200.0$, and 6000.0 , respectively. We used the median value as a threshold to classify the farm size because the data was not normal distribution. The result of the multiple logistic regression to assess the interaction between variables including the mean distance to the closest farm in multiple logistic regression analysis is summarized in Table 2.
$C I$ confidence intervals, $F F$ farrow-to-finish production type, $F W$ farrow-to-wean production type, $W F$ wean-tofinish production type

Geographical location mapping of all farms using QGIS showed that PEDV-positive farms were distributed throughout the study area (Fig. 1b). The summary distance of geographical data of all farms including mean, standard deviation, min, and max values were $0.85,0.46,0.01$, and $3.4 \mathrm{~km}$, respectively. The nearest neighbor index was 0.44 (less than 1) and the $\mathrm{Z}$ score value was $-19.37(P<0.01)$. This reflected a high degree of clustering of swine farms.

\section{Discussion}

To the best of our knowledge, these findings are the first to highlight the following two points and thus contribute to the further understanding of PEDV. First, the proportion of PEDV-positive pig farms in Vietnam was $30.9 \%$ in highdensity pig population areas over the study period. Second, herd size $(\geq 600)$ and area (Northeast and Red River Delta) were considered to be risk factors for PEDV spread in Vietnam.

Our study represents the first cross-sectional PEDV survey carried out in Vietnam to evaluate the proportion of PEDVpositive pig farms and capture the basic epidemiologic features of PED. The high proportion of PEDV-positive farms in Vietnam is an important baseline epidemiological feature that could provide a useful comparison with future findings. Most piglets are produced in the northern Thai Binh, Hung Yen, Bac Giang, and Ha Nam provinces in the Northeast and Red River Delta, and then pigs are transported to other mountainous areas and to southern Vietnam (Dietze 2011). Therefore, the high proportion of PEDV-positive farms in northern and central Vietnam could be a potential source of nationwide PEDV transmission. The proportion of PEDV-positive farms in Vietnam was higher than that in the US and Japan (27.9\% and $19.5 \%$, respectively) (Alvarez et al. 2016; Sasaki et al. 2017) where syndromic surveillance has been carried out to help farm owners respond to PED outbreaks (United States Department of Agriculture and Service 2014; Ministry of Agriculture Forestry and Fisheries (MAFF) 2016). The results of a previous study provided evidence of the presence of seropositive animals on PEDV-negative farms (Koike et al. 2018). In this study, PEDV was detected on $30.89 \%$ (101/ 327) of farms during the study period. In 101 PEDVpositive farms, $28.71 \%$ (29/101) of farms were affected by PED and had animals showing clinical signs such as severe watery diarrhea with high morbidity and mortality. More than $70 \%$ of PEDV-positive farms had subclinically infected animals, which could increase the risk of cross-contamination. However, since the initial detection of PEDV in Vietnam in 2009 , no specific biosecurity measures have yet been applied 
Table 1 Proportion of PEDVpositive pig farms in Vietnam from January 2018 to February 2019

\begin{tabular}{llllr}
\hline Variable & $\begin{array}{l}\text { Number of } \\
\text { collected farms }\end{array}$ & $\begin{array}{l}\text { Number of } \\
\text { PED-positive farms }\end{array}$ & $\begin{array}{l}\text { Proportion of } \\
\text { PED-positive farms }\end{array}$ & 95\% CI \\
\hline Farm status & & & & \\
Companies & 197 & 68 & 34.5 & $27.9-41.6$ \\
Private & 130 & 33 & 25.4 & $18.2-33.8$ \\
Production type & & & & \\
FF & 185 & 57 & 30.8 & $24.2-38.0$ \\
FW & 88 & 21 & $23.9^{\mathrm{a}}$ & $15.4-34.1$ \\
Nucleus & 10 & 7 & $70.0^{\mathrm{b}}$ & $34.8-93.3$ \\
WF & 44 & 16 & 36.4 & $22.4-52.2$ \\
Area & & & & $23.6-51.0$ \\
Northeast & 52 & 19 & $36.5^{\mathrm{a}}$ & $6.0-61.0$ \\
Northwest & 11 & 3 & 27.3 & $27.4-40.3$ \\
Red River Delta & 220 & 74 & $33.6^{\mathrm{a}}$ & $3.8-24.6$ \\
North central coast & 44 & 5 & $11.4^{\mathrm{b}}$ & \\
Size & & & & $23.8^{\mathrm{a}}$ \\
$<600$ & 160 & 38 & $37.7^{\mathrm{b}}$ & $30.4-45.5$ \\
\hline 600 & 167 & 63 & &
\end{tabular}

Values with different letters are significantly different from the others $(P<0.05)$ to prevent the spread of the virus. During current Vietnamese PED outbreaks, poor biosecurity procedures related to fomites, animals, and humans could be the major risk factors underlying nationwide PEDV transmission. Biosecurity practices such as using different trucks to carry pigs from different farms based on PEDV infection status, improving in farm hygiene management and avoiding risky practices associated with contact with pig excrement, were done to prevent the spreading of PEDV in Japan and US (United States Department of Agriculture and Service 2014; Koike et al. 2018). Our results could contribute to producers to implement similar biosecurity measures to prevent the further spreading of PEDV in Vietnam.

The higher proportion of PEDV-positive farms observed in the Northeast and Red River Delta areas containing a higher density of pig populations was consistent with previous studies that also indicated that the highest proportion of PEDVpositive farms was found in high-density pig production areas (Alvarez et al. 2016; Boniotti et al. 2018). Some northern provinces in the Northeast and Red River Delta areas are the main areas of intensive pig production in northern Vietnam and constitute prime locations for porcine diseases, including emergence of highly virulent porcine reproductive and respiratory syndrome (PRRS) (Dietze 2011; Lee et al. 2019). Recently, African swine fever (ASF) was also first detected in the Red River Delta (Hung Yen and Thai Binh provinces) on 19 February 2019 (Le et al. 2019) and quickly spread to 24 provinces in the Red River Delta, Northeast, Northwest, and North Central Coast regions (Food and Agriculture Organization 2019). Therefore, it is necessary to detect PEDV-positive farms and implement strict biosecurity measures to prevent spread of PEDV to other pig production regions in Vietnam such as dividing truck for pig carrying assigning from different farms based on their PEDV infection status to prevent the spread of PEDV between farms.

In this study, the nearest neighbor index was 0.44 and there was a high degree of clustering of swine farms. Airborne spread of PEDV among neighboring farms due to movement of animals, humans, and contaminated vehicles was demonstrated to be a potential mechanism of transmission (Do et al.
Table 2 Result of multiple logistic regression analysis to assess the interaction between variables and PEDV pig farm status in Vietnam from January 2018 to February 2019

\begin{tabular}{lccccc}
\hline Variables & Coefficient & SE & $\begin{array}{l}\text { Z- } \\
\text { statistic }\end{array}$ & $P$ value & OR (95\% CI) \\
\hline Intercept & 0.9214 & 0.4894 & 1.883 & 0.05 & \\
Size $(<600)$ & -0.7455 & 0.2498 & -2.985 & 0.00284 & $1.94(1.20-3.14)$ \\
Area (Northeast and Red River Delta) & -1.22 & 0.4087 & -2.985 & 0.00283 & $3.05(1.38-6.73)$ \\
Mean distance & -0.2242 & 0.4756 & -0.471 & 0.673 & \\
\hline
\end{tabular}


2011; Beam et al. 2015). Distance from the closest PEDpositive farm $(<1000 \mathrm{~m})$ was indicated as a risk factor for PEDV transmission (Alonso et al. 2014; Madson et al. 2014; Beam et al. 2015; Sasaki et al. 2016). Strict biosecurity measures are very effective in protecting susceptible pigs, but since the initial detection of PEDV in Vietnam in 2009, no air treatments by spraying disinfectant water for the surrounding environment of PED-positive farms have been conducted in areas with a high density of pig farms, which could increase the likelihood of PEDV survival and transmission. As a result, PEDV-positive farms were distributed throughout the study area. PED is known to result in significant economic losses due to the high morbidity and mortality in neonatal piglets. An average of 2.7 piglets/sow were not weaned and the mean time required to regain baseline production was 10 weeks (Goede and Morrison 2016). PED has also been indicated to cause reductions in the number weaned and increased abortions in sows (Scanlan et al. 2019). For the finishing farms, there was an increase in mortality and feed conversion ratio and a decrease of average daily gain in PEDV-infected farms (Alvarez et al. 2015; Schweer et al. 2016). It is necessary to identify PEDV-positive farms and implement thorough biosecurity management procedures to reduce economic losses due to PEDV infection including cleaning and disinfection of pig houses and surrounding environment.

In previous studies, PED farm-level prevalence was higher on larger farms than on smaller farms (Carvajal et al. 1995; Sasaki et al. 2017). In our study, the proportion of PEDVpositive farms was also higher for larger farms. This finding could be related to an increased number of contacts by a larger number of staff and more frequent visits of trucks to larger farms, making these farms more susceptible to PED outbreaks in comparison with smaller farms. The number of transport vehicles visiting a farm for pigs and feed has been previously reported as a potential means of spread for PEDV (Lowe et al. 2014; Sasaki et al. 2016). Moreover, breeding sows are replaced more frequently on large farms than on small farms. A previous study demonstrated that PEDV can persist on a farm after an initial outbreak if there is a continuous input of susceptible animals (Pijpers et al. 1993). This could lead to PEDV-positive large farms remaining in a vicious circle in which PED outbreaks continue to recur. However, large farms should be considered at increased risk and should maximize their biosecurity measures in case of a PED outbreak in the area by personnel and vehicle restriction.

Farms of the nucleus production type showed the highest proportion of PEDV-positivity in northern Vietnam. Firstly, there is a possibility that the nucleus production type introduces their GGP or GP pigs from China or other countries, and their pigs were infected with PEDV. However, in fact, the nucleus farms introduce pigs from other countries without the PEDV quarantine certificate. PEDV was considered to be introduced in feeder pigs, fattening pigs, and adult pigs, and then spread to piglets (Pensaert and Martelli 2016; Wang et al. 2016). In addition, after the first epidemic period of PED, PEDV often existed in weaned and feeder pigs of breedingfinishing herds (Pijpers et al. 1993). The number of sows in a farm was suspected to play a role in the persistence of PEDV after the original outbreak (Pensaert and Martelli 2016). Based on the above reasons, the proportion of PEDV-positive farms among nucleus production type farms was significantly higher than other production types. Therefore, sows in nucleus production type farms could be a potential risk for PEDV spread to other pigs of different ages as well as farms of other production types. A higher proportion of PEDV-positivity on farms where sows were present was demonstrated in other previous studies in the US (Beam et al. 2015; Alvarez et al. 2016). Moreover, all nucleus farms in this study were large farms and located in areas with higher risk of PEDV infection with higher pig density and in the main areas of pig production in the Northeast and Red River Delta. It is therefore critical for PEDV-positive farms to further tighten biosecurity measures to prevent cross-contamination between production types, especially for farms of the nucleus production type including cleaning and disinfection of pig houses and surrounding environment, changing clothes and boots of workers and personnel and vehicle restriction.

This study had the limitation that samples were collected from fewer farms located in mountainous areas, and no samples were collected from farms located in southern Vietnam. In addition, though SADS-CoV and TGEV have not been detected so far, there remains the possibility that other enteric viruses may be identified if we could perform the appropriate tests. Therefore, it is important to improve national surveillance data as well as sample collection and management systems. Further molecular studies including PEDV sequencing data are required to understand the risk factors associated with PEDV transmission in Vietnam.

In conclusion, this is the first report of the high proportion of PEDV-positive farms and the epidemiology of PED in northern Vietnam by using the pooled testing system to identify PEDV-infected farms. PEDV was detected in $30.89 \%$ $(101 / 327)$ of farms during the study period. More than $70 \%$ of PEDV positive farms contained animals that were subclinically infected, which could increase the risk of cross-contamination. Prevention and control strategies should thus be implemented to limit the spread of PEDV in the Vietnamese swine industry. Our data contribute to further elucidation of the epidemiological dynamics of PEDV infection, providing knowledge to establish realistic objectives for the prevention and/or control of this disease.

Acknowledgments We thank all veterinarians for help in collecting fecal samples. We also thank the pig producers in Vietnam who participated in this study. We thank Gillian Campbell, PhD, from Edanz Group (www. edanzediting.com/ac) for editing a draft of this manuscript. 
Author contributions Conceptualization, SS; collecting samples, TNM and TPB; methodology, WY; CFI-RT-LAMP experiments, TNM; data analysis, TNM and SS; writing - original draft preparation, TNM; writing - review and editing, SS; supervision, review and editing, SS, JN, VGN, TMLH, SM, HD, and EK; support for study design, WY and $J N$. All authors have read and agreed to the final version of the manuscript.

Funding This work was supported by the Ministry of Education, Culture, Sports, Science and Technology (MEXT) and Japan Society for the Promotion of Science, Grants-in-Aid for Scientific Research - JSPS KAKENHI (grant number JP15K18786).

\section{Compliance with ethical standards}

Conflict of interest None of the authors of this paper have a financial or personal relationship with other people or organizations that could inappropriately influence or bias the content of the paper. Author Bui Thanh Phong was employed by the company Masan Nutri-Science, Masan Group. The remaining authors declare that the research was conducted in the absence of any commercial or financial relationships that could be construed as a potential conflict of interest. The funders had no role in the design of the study, in the collection, analyses, or interpretation of the data, in the writing of the manuscript, or in the decision to publish the results.

Ethics approval All participating farms did so voluntarily, and verbal consent was obtained from veterinarians, farm managers, or farm owners. All samples were collected in accordance with the procedures approved by the Animal Ethics Committee of the University of Miyazaki's Faculty of Agriculture (Number 2017-541) and by the Hanoi School of Public Health Institutional Review Board (HSPHIRB), approval number 019405/DD-YTCC.

Availability of data and material The datasets supporting the conclusions of this article are included within the article. All datasets are available from the corresponding author on reasonable request.

\section{References}

Alonso, C., Goede, D.P., Morrison, R.B., Davies, P.R., Rovira, A., Marthaler, D.G. and Torremorell, M., 2014. Evidence of infectivity of airborne porcine epidemic diarrhea virus and detection of airborne viral RNA at long distances from infected herds, Veterinary Research, 45: 73.

Alvarez, J., Sarradell, J., Morrison, R. and Perez, A., 2015. Impact of porcine epidemic diarrhea on performance of growing pigs, PLoS One, 10: e0120532.

Alvarez, J., Goede, D., Morrison, R. and Perez, A., 2016. Spatial and temporal epidemiology of porcine epidemic diarrhea (PED) in the Midwest and Southeast regions of the United States, Prev Vet Med, 123: $155-160$.

Beam, A., Goede, D., Fox, A., McCool, M.J., Wall, G., Haley, C. and Morrison, R., 2015. A Porcine Epidemic Diarrhea Virus Outbreak in One Geographic Region of the United States: Descriptive Epidemiology and Investigation of the Possibility of Airborne Virus Spread, PLoS One, 10: e0144818.

Boniotti, M.B., Papetti, A., Bertasio, C., Giacomini, E., Lazzaro, M., Cerioli, M., Faccini, S., Bonilauri, P., Vezzoli, F., Lavazza, A. and Alborali, G.L., 2018. Porcine Epidemic Diarrhoea Virus in Italy: Disease spread and the role of transportation, Transbound Emerg Dis, 65: 1935-1942.
Carvajal, A., Lanza, I., Diego, R., Rubio, P. and Cármenes, P., 1995. Seroprevalence of porcine epidemic diarrhea virus infection among different types of breeding swine farms in Spain, Preventive Veterinary Medicine, 23: 33-40.

Chen, X., Zhang, X.X., Li, C., Wang, H., Wang, H., Meng, X.Z., Ma, J., Ni, H.B., Zhang, X., Qi, Y. and Sun, D., 2019. Epidemiology of porcine epidemic diarrhea virus among Chinese pig populations: A meta-analysis, Microbial Pathogenesis, 129: 43-49.

Choe, S.E., Park, K.H., Lim, S.I., Le, V.P., Hien, N.B., Thach, P.N., Phuong le, H.T., An, B.H., Han, S.H., Cho, I.S. and An, D.J., 2016. Complete Genome Sequence of a Porcine Epidemic Diarrhea Virus Strain from Vietnam, HUA-14PED96, with a Large Genomic Deletion, Genome Announc, 4.

Diep, N.V., Sueyoshi, M., Izzati, U., Fuke, N., Teh, A.P.P., Lan, N.T. and Yamaguchi, R., 2018. Appearance of US-like porcine epidemic diarrhoea virus (PEDV) strains before US outbreaks and genetic heterogeneity of PEDVs collected in Northern Vietnam during 20122015, Transbound Emerg Dis, 65: e83-e93.

Dietze, K., 2011. Porcine reproductive and respiratory syndrome (PRRS): virulence jumps and persistent circulation in Southeast Asia, Focus on ..., 5: 8 .

Do, T.D., Nguyen, T.T., Suphasawatt, P. and Roongroje, T., 2011. Genetic characterization of porcine epidemic diarrhea virus (PEDV) isolates from Southern Vietnam during 2009-2010 outbreaks, Thai J Vet Med, 41: 55-64.

Food and Agriculture Organization. ASF situation in Asia update. 2019. http://www.fao.org/ag/againfo/programmes/en/empres/ASF/ Situation update.html. Accessed 2nd May 2019

Goede, D. and Morrison, R.B., 2016. Production impact \&amp; time to stability in sow herds infected with porcine epidemic diarrhea virus (PEDV), Preventive Veterinary Medicine, 123: 202-207.

Hayashida, K., Kajino K, Hachaambwa L, Namangala B and Sugimoto C, 2015. Direct Blood Dry LAMP: A Rapid, Stable, and Easy Diagnostic Tool for Human African Trypanosomiasis, PLOS Neglected Tropical Diseases, 9: e0003578.

Kim, Y.K., Lim, S.I., Lim, J.A., Cho, I.S., Park, E.H., Le, V.P., Hien, N.B., Thach, P.N., Quynh do, H., Vui, T.Q., Tien, N.T. and An, D.J., 2015. A novel strain of porcine epidemic diarrhea virus in Vietnamese pigs, Arch Virol, 160: 1573-1577.

Kochhar, H.S., 2014. Canada: Porcine epidemic diarrhea in Canada: an emerging disease case study, Can Vet J, 55: 1048-1049.

Koike, N., Mai, T.N., Shirai, M., Kubo, M., Hata, K., Marumoto, N., Watanabe, S., Sasaki, Y., Mitoma, S., Notsu, K., Okabayashi, T., Wiratsudakul, A., Kabali, E., Norimine, J. and Sekiguchi, S., 2018. Detection of neutralizing antibody against porcine epidemic diarrhea virus in subclinically infected finishing pigs, J Vet Med Sci, 80: 1782-1786.

Le, V.P., Dae Gwin, J., Sun-Woo, Y., Hye-Min, K., Thi Bich Ngoc, T., Thi Lan, N., Thi To Nga, B., Jinsik, O., Joon Bae, K., Kwang Myun, C., Nguyen Van, T., Eunhye, B., Thi Thu Hang, V., Minjoo, Y., Woonsung, N. and Daesub, S., 2019. Outbreak of African Swine Fever, Vietnam, 2019, Emerging Infectious Diseases, 25.

Lee, C., 2015. Porcine epidemic diarrhea virus: An emerging and reemerging epizootic swine virus, Virology Journal, 12: 193-193.

Lee, H.S., Pham, T.L., Nguyen, T.N., Lee, M. and Wieland, B., 2019. Seasonal patterns and space-time clustering of porcine reproductive and respiratory syndrome (PRRS) cases from 2008 to 2016 in Vietnam, Transboundary and Emerging Diseases, 66: 986-994.

Lin, C.N., Chung, W.B., Chang, S.W., Wen, C.C., Liu, H., Chien, C.H. and Chiou, M.T., 2014. US-like strain of porcine epidemic diarrhea virus outbreaks in Taiwan, 2013-2014, J Vet Med Sci, 76: 12971299.

Lowe, J., Gauger, P., Harmon, K., Zhang, J., Connor, J., Yeske, P., Loula, T., Levis, I., Dufresne, L. and Main, R., 2014. Role of transportation in spread of porcine epidemic diarrhea virus infection, United States, Emerging Infectious Diseases, 20: 872-874. 
Madson, D.M., Magstadt, D.R., Arruda, P.H., Hoang, H., Sun, D., Bower, L.P., Bhandari, M., Burrough, E.R., Gauger, P.C., Pillatzki, A.E., Stevenson, G.W., Wilberts, B.L., Brodie, J., Harmon, K.M., Wang, C., Main, R.G., Zhang, J. and Yoon, K.J., 2014. Pathogenesis of porcine epidemic diarrhea virus isolate (US/ Iowa/18984/2013) in 3-week-old weaned pigs, Veterinary Microbiology, 174: 60-68.

Mai, T., Nguyen VD, Yamazaki W, Okabayashi T, Mitoma S, Notsu K, Sakai Y, Yamaguchi R, Norimine J and Sekiguchi S, 2018. Development of pooled testing system for porcine epidemic diarrhoea using real-time fluorescent reverse-transcription loop-mediated isothermal amplification assay, BMC Veterinary Research, 14: 172.

Ministry of Agriculture Forestry and Fisheries (MAFF), J. Porcine epidemic diarrhea. 2016. http://www.maff.go.jp/j/syouan/douei/ped/ ped.html Accessed 27th May 2017

Niederwerder, M.C. and Hesse, R.A., 2018. Swine enteric coronavirus disease: A review of 4 years with porcine epidemic diarrhoea virus and porcine deltacoronavirus in the United States and Canada, Transbound Emerg Dis, 65: 660-675.

Pensaert, M.B. and De Bouck, P., 1978. A new coronavirus-like particle associated with diarrhea in swine, Archives of Virology, 58: 243247.

Pensaert, M.B. and Martelli, P., 2016. Porcine epidemic diarrhea: A retrospect from Europe and matters of debate, Virus Res, 226: 1-6.

Pijpers, A., van Nieuwstadt, A.P., Terpstra, C. and Verheijden, J.H., 1993. Porcine epidemic diarrhoea virus as a cause of persistent diarrhoea in a herd of breeding and finishing pigs, Veterinary Record, 132: 129-131.

Sasaki, Y., Alvarez, J, Sekiguchi, S, Sueyoshi, M, Otake, S and Perez, A., 2016. Epidemiological factors associated to spread of porcine epidemic diarrhea in Japan, Prev Vet Med, 123: 161-167.

Sasaki, Y., Toyomaki, H., Sekiguchi, S., Sueyoshi, M., Makita, K., Otake, S., Perez, A. and Alvarez, J., 2017. Spatial dynamics of porcine epidemic diarrhea (PED) spread in the southern Kyushu, Japan, Prev Vet Med, 144: 81-88.

Scanlan, C.L., Putz, A.M., Gray, K.A. and Serão, N.V.L., 2019. Genetic analysis of reproductive performance in sows during porcine reproductive and respiratory syndrome (PRRS) and porcine epidemic diarrhea (PED) outbreaks, Journal of Animal Science and Biotechnology, 10: 22.
Schweer, W.P., Schwartz, K., Burrough, E.R., Yoon, K.J., Sparks, J.C. and Gabler, N.K., 2016. The effect of porcine reproductive and respiratory syndrome virus and porcine epidemic diarrhea virus challenge on growing pigs I: Growth performance and digestibility1, Journal of Animal Science, 94: 514-522.

Song, D. and Park, B., 2012. Porcine epidemic diarrhoea virus: a comprehensive review of molecular epidemiology, diagnosis, and vaccines, Virus Genes, 44: 167-175.

Takahashi, K., Okada, K. and Ohshima, K., 1983. An outbreak of swine diarrhea of a new-type associated with coronavirus-like particles in Japan, Journal of Veterinary Medical Science, 45: 829-832.

Thomas, P.R., Karriker, L.A., Ramirez, A., Zhang, J., Ellingson, J.S., Crawford, K.K., Bates, J.L., Hammen, K.J. and Holtkamp, D.J., 2015. Evaluation of time and temperature sufficient to inactivate porcine epidemic diarrhea virus in swine feces on metal surfaces, Journal of Swine Health and Production, 23: 84.

Toyomaki, H., Sekiguchi, S., Sasaki, Y., Sueyoshi, M. and Makita, K., 2018. Factors associated with farm-level infection of porcine epidemic diarrhea during the early phase of the epidemic in Japan in 2013 and 2014, Preventive Veterinary Medicine, 150: 77-85.

United States Department of Agriculture and Service, A.a.P.H.I. Herd Monitoring and Management Plan for Novel Swine Enteric Coronavirus Diseases. 2014. https://www.aphis.usda.gov/animal health/animal_dis_spec/swine/downloads/secd_monitoring management plan.pdf. Accessed 5th June 2014

Vui, D.T., Tung, N., Inui, K., Slater, S. and Nilubol, D., 2014. Complete genome sequence of porcine epidemic diarrhea virus in Vietnam, Genome Announc, 2.

Vui, D.T., Thanh, T.L., Tung, N., Srijangwad, A., Tripipat, T., Chuanasa, T. and Nilubol, D., 2015. Complete genome characterization of porcine epidemic diarrhea virus in Vietnam, Arch Virol, 160: 1931-1938.

Wang, D., Fang, L. and Xiao, S., 2016. Porcine epidemic diarrhea in China, Virus Res, 226: 7-13.

Wood, E., 1977. An apparently new syndrome of porcine epidemic diarrhoea, Veterinary Record, 100: 243-244.

Publisher's note Springer Nature remains neutral with regard to jurisdictional claims in published maps and institutional affiliations. 